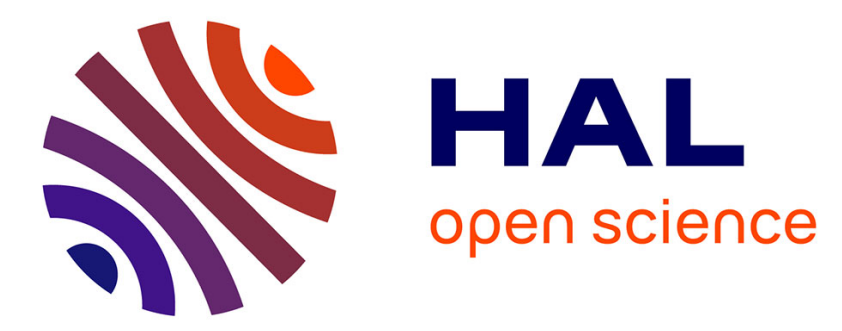

\title{
High-contrast 10 fs OPCPA-based front end for multi-PW laser chains
}

\author{
N. Papadopoulos, P. Ramirez, K. Genevrier, L. Ranc, N. Lebas, A. Pellegrina, \\ C. Le Blanc, P. Monot, L. Martin, P. Zou, et al.
}

\section{- To cite this version:}

N. Papadopoulos, P. Ramirez, K. Genevrier, L. Ranc, N. Lebas, et al.. High-contrast 10 fs OPCPA-based front end for multi-PW laser chains. Optics Letters, 2017, 42 (18), pp.3530-3533. 10.1364/OL.42.003530 . hal-01676598

\section{HAL Id: hal-01676598 \\ https://hal-iogs.archives-ouvertes.fr/hal-01676598}

Submitted on 8 Jan 2018

HAL is a multi-disciplinary open access archive for the deposit and dissemination of scientific research documents, whether they are published or not. The documents may come from teaching and research institutions in France or abroad, or from public or private research centers.
L'archive ouverte pluridisciplinaire HAL, est destinée au dépôt et à la diffusion de documents scientifiques de niveau recherche, publiés ou non, émanant des établissements d'enseignement et de recherche français ou étrangers, des laboratoires publics ou privés. 


\title{
High-contrast 10-fs OPCPA-based front-end for multi- PW laser chains
}

\author{
D. N. Papadopoulos ${ }^{1 *}$, P. Ramirez ${ }^{1,2}$, K. GeneVRIER ${ }^{1,2}$, L. RANC ${ }^{1}$, N. Lebas $^{1}$, \\ A. Pellegrina ${ }^{1}$, C. Le Blanc ${ }^{1}$, P. MOnOt $^{3}$, L. Martin ${ }^{1}$, J.P. ZOu ${ }^{1}$, \\ F. Mathieu ${ }^{1}$, P. Audebert ${ }^{1}$, P. Georges ${ }^{2}$, F. Druon ${ }^{*}$ \\ ${ }^{1}$ Laboratoire pour I'Utilisation des Lasers Intenses, CNRS, Ecole Polytechnique, Palaiseau, France \\ ${ }^{2}$ Laboratoire Charles Fabry, Institut d'Optique Graduate School, CNRS, Université Paris-Saclay, 91127 Palaiseau cedex, France \\ ${ }^{3}$ CEA, Iramis, SPAM, Saclay, France \\ *Corresponding authors: frederic.druon@institutoptique.fr, dimitrios.papadopoulos@polytechnique.edu.
}

Received XX Month XXXX; revised XX Month, XXXX; accepted XX Month XXXX; posted XX Month XXXX (Doc. ID XXXXX); published XX Month XXXX

\begin{abstract}
Applications using multi-PW lasers necessitate high temporal pulse quality with a tremendous contrast ratio. The first crucial prerequisite, to achieve multi-PW peak power, is the generation of ultra-short pulses with good spectral phase quality. Second, to avoid any deleterious pre-ionisation effect on targets, ns-contrast better than $10^{12}$ is also targeted. In the framework of the Apollon 10PW French laser program, we present a high-contrast 10fs Front-End design study to inject high energetic Ti:sapphire $\mathrm{PW}$-lasers. The contrast ratio has been measured and analyzed in different time ranges highlighting the different major contributions for each scale.
\end{abstract}

OCIS codes: Lasers titanium, Ultrafast lasers, Ultrafast nonlinear optics.

http://dx.doi.org/10.1364/OL.99.099999

Multi-PW laser projects flourish all over the world [1]. One of the main issues for the applications linked to these ultra-highintense systems concerns the temporal pulse quality and in particular the contrast in the front of the pulses [1-5]. Indeed, at such level of intensity, unwanted interaction [6] with targets and plasma preformation easily occurs necessitating nscontrast better than $10^{-12}$. Moreover, short pulses of high quality and minimal residual spectral phase are also an important prerequisite in order to permit an optimal temporal localization of the energy. In the framework of the Apollon 10PW French laser program, a high-contrast 10-fs Front-End for Ti:Sapphire PW-lasers has been developed. The Apollon laser, currently under construction at the University of Paris-Saclay, France, will be among the first multi-PW installations in the world devoted to the study of high intensity laser matter interaction at unprecedented regimes and peak intensities surpassing $2 \cdot 10^{22} \mathrm{~W} / \mathrm{cm}^{2}$. The final goal of the Apollon laser is the generation of $10 \mathrm{PW}$ pulses corresponding to energy of $150 \mathrm{~J}$ and $15 \mathrm{fs}$ duration at a repetition rate of 1 shot/minute. To fulfill the objectives, Apollon is based on a so called "hybrid" configuration where the optical parametric chirped pulse amplification (OPCPA) technology is optimally combined with Ti:Sapphire based main amplification [7]. OPCPA in the few picosecond regime is used in the Front End of the system to guarantee a large bandwidth and high contrast injection pulses of about $1 \mathrm{~mJ}$ at $100 \mathrm{~Hz}$ repetition rate.

In this work we present a review of earlier and more recently obtained results from the OPCPA stage of this front-end source. The design considerations and the parametric optimization of the laser source configuration are discussed in detail taking into account the parametric conversion efficiency, the saturation level, the spatial aspects and finally the impact on the contrast ratio in different time scales. The contrast ratio analysis considers the fs range for the coherent contrast issues, the $10 \mathrm{~s}$ ps range for close non-coherent issues, and the $100 \mathrm{~s}-\mathrm{ps}$ range for fluorescence issues. Major impacting effects on each scale are described and discussed concluding to noteworthy results especially in the $10 \mathrm{~s}$ ps range.

As summarized in fig. 1, the front-end system is based on an optically-synchronous OPCPA operating in the few picosecond range. An ultrashort Ti:Sapphire oscillator ( $<7 \mathrm{fs}$ ) produces a very broad spectrum that is split into two outputs : 1) the main part centered at $800 \mathrm{~nm}$ is sent to a Ti:Sapphire multipass amplifier that generates $>1.5 \mathrm{~mJ}, 25 \mathrm{fs}$ pulses at $1 \mathrm{kHz}$ with a relatively high contrast ratio (CR) of $\sim 10^{-8}$ and 2 ) the spectral part $>1020 \mathrm{~nm}$ is used to inject an Yb-based chirped pulse amplification (CPA) system that is used to pump the OPCPA.

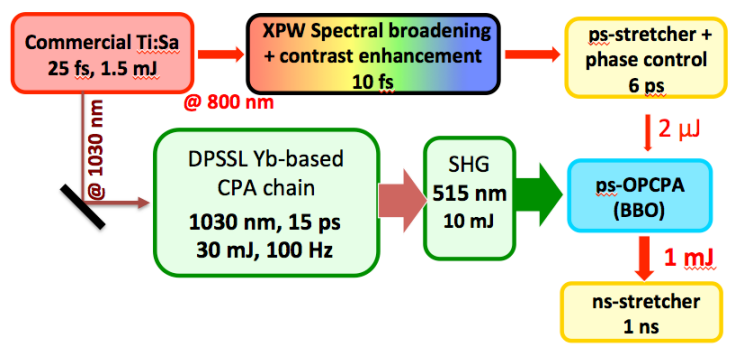

Figure 1: Apollon front-end experimental scheme. 


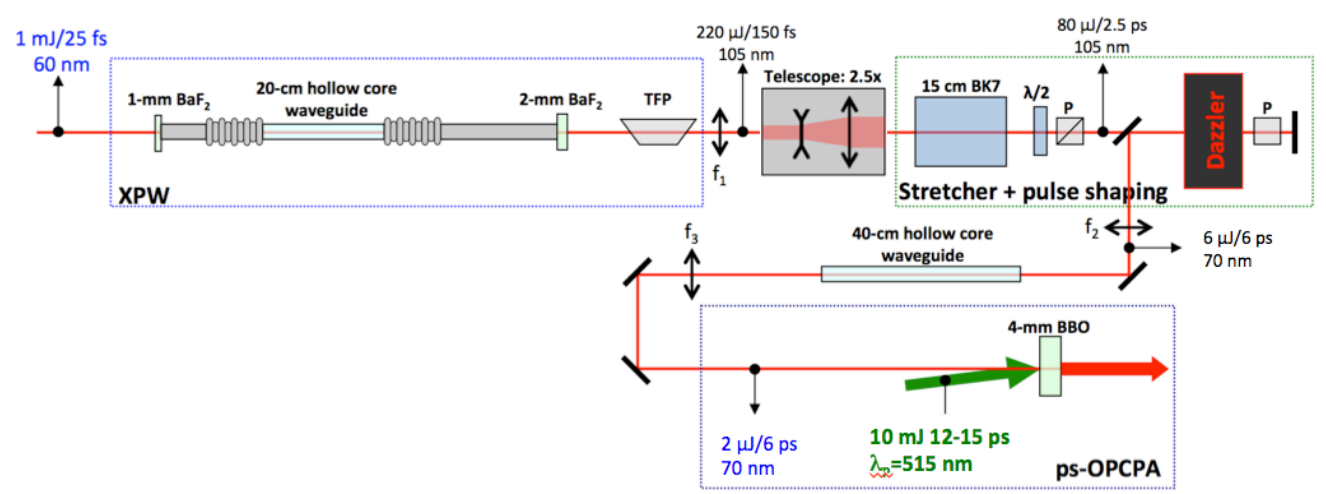

Figure 2: Experimental setup of the contrast-cleaner, phase-and-spectrum-controller apparatus of the Apollon 10 PW laser. (TFP for thin film polarizer)

The operation point of the OPCPA stage is chosen on the basis of three main considerations: First, to provide a relatively low saturated gain to limit the generation of unwanted superfluorescence and at the same time improve the CR of the pulses (in the time range of the pump pulse duration) by the same factor, 2); second, to provide output energy in the $1 \mathrm{~mJ}$ range to guarantee the comfortable injection of the following amplification stages based on Ti:Sapphire, without excessive requirements on the OPCPA pump pulse energy limited around $10 \mathrm{~mJ}$ (at $515 \mathrm{~nm}$ ); last, to operate on a controllable, moderate saturation level to guarantee the combination of stable operation and good beam quality.

In fig. 2 is presented a more detailed schematic of the OPCPA injection setup. The input signal is provided by a double stage XPW (cross-polarized wave) configuration which plays a double role: the improvement of the contrast of the commercial CPA source by at least $2-3$ orders of magnitude ( $\mathrm{CRxPW}<10^{-10}$ ), and the spectral broadening and shaping providing a Gaussian like spectrum supporting sub-10 fs pulses [9-11]. The signal is then stretched to $\sim 6$ ps in a $15 \mathrm{~cm}$ block of BK7 and goes through a high dynamic range and high spectral phase correction capacity active controller, a double-pass acoustooptic programmable dispersive filter (AOPDF, Dazzler from Fastlite) employing a 45-mm long $\mathrm{TeO}_{2}$ crystal. This device will be used to compensate the spectral phase distortions of the entire laser chain. The beam is then spatially filtered with a 40$\mathrm{cm}$ long hollow core waveguide (HCW) before being sent into the OPCPA crystal. Spatial filtering with a HCW is used to guarantee a stable filtering function and a fixed beam pointing reference for the rest of the laser chain. Due to the reduced efficiency of the double pass AOPDF and the strong degradation of the beam observed after it (fig. 3), the OPCPA seed energy is decreased to $\sim 2 \mu \mathrm{J}$. The final beam profile however is excellent and the energy stability is $<1.6 \% \mathrm{rms}$ for an input laser stability of $<1.25 \% \mathrm{rms}$ (over $30 \mathrm{~min}$ ).
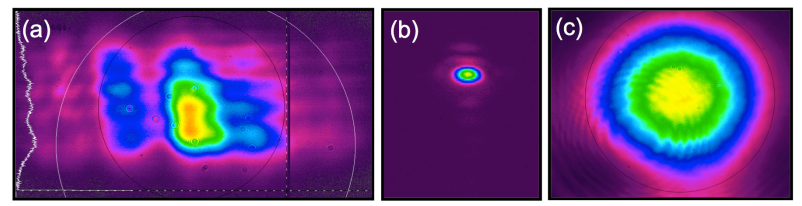

Figure 3: Beam profiles of the stretched seed: (a) near field after the AOPDF, (b) far field at the HCW filter, (c) near field at the OPCPA input.

Regarding the OPCPA pump beam, the $1030 \mathrm{~nm}$ output of the oscillator is first amplified in an Yb-doped fiber amplifier (from Keopsys), stretched to $1.5 \mathrm{~ns}$, and amplified to $2 \mathrm{~mJ}$ in a commercial $\mathrm{Yb}: \mathrm{KYW}$ regenerative amplifier (from Amplitude Systemes). According to our design a final booster of the pump signal to reach the energy level of $<30 \mathrm{~mJ}$ is then required to provide the $10 \mathrm{~mJ}$ pulse at $515 \mathrm{~nm}$ (for typical compression $(\sim 70 \%)$ and frequency doubling ( $\sim 60 \%)$ efficiencies). In order to access to a larger range of experimental conditions and better investigate the limitations imposed by the saturation effects, we decided to perform the validating experiments using a pump with higher energy. A Yb:YAG thin-disk regenerative amplifier capable of delivering up to $150 \mathrm{~mJ}$ at $100 \mathrm{~Hz}$ [11] is then used. The output of the final amplifier is compressed down to $\sim 15 \mathrm{ps}$ and frequency-doubled in a $4 \mathrm{~mm}$ thick type-I LBO crystal producing approximately 12 ps pulses at $515 \mathrm{~nm}$ with adjustable energy up to $60 \mathrm{~mJ}$.

The OPCPA nonlinear crystal is a $4 \mathrm{~mm}$ thick BBO cut for type-I interaction at $24.5^{\circ}$ phase matching angle. The input beam is collimated at 5-mm-diameter with the use of single concave mirror at the output of the filtering HCW. The beam size and the energy of the pump beam could be varied (with the use of several lens combinations) to allow the parametric study and the optimization of the operation of the amplifier [14]. The goal is to obtain a stage which when pumped with 10-11 mJ, would deliver a stable amplified signal, with an energy around $1 \mathrm{~mJ}$, bandwidth for 10 -fs pulses and an excellent beam profile. The main challenge in this parametric investigation is to achieve a well-controlled saturation level. Saturation guarantees both increased stability and efficient energy transfer. It should however be limited to the optimal level so that deleterious back-conversion effects leading to degradation of the spatial and the spectral form of the amplified pulses are not significant. The optimal pump diameter has been defined to be around 3 $\mathrm{mm}\left(1 / \mathrm{e}^{2}\right)$ with a Gaussian like shape. The choice of using a larger seed beam has been favored as a precaution based on a method to suppress super-fluorescence implemented in [15], where the larger signal beam allows the selection of the part of the signal beam with the highest signal to noise ratio and cleanest wavefront. The behavior of the OPCPA is therefore investigated with respect to the pump beam energy, which leads to intensities ranging from $3.8-15.1 \mathrm{GW} / \mathrm{cm}^{2}$.

Under perfect phase matching conditions, the injected spectrum with a Gaussian like shape and a FWHM of 70-nm (reduction due to the AOPDF response which is not perfectly flat in the whole $200 \mathrm{~nm}$ spectral range), is broadened and progressively reaches a square-shaped form for increasing pump energy (fig. 4 - left graph). The spectrum converges to a square shape when the pump energy exceeds $9 \mathrm{~mJ}$, which corresponds to the saturation level also well observed on the efficiency. Above $10 \mathrm{~mJ}$ of pump, the spectrum still enlarges up to $200 \mathrm{~nm}$ and the energy exceeds $1 \mathrm{~mJ}$. Nevertheless, at this point the saturation begins to be deleterious on the beam profile and a rollover is observed also on the efficiency (fig. 4 right side). 

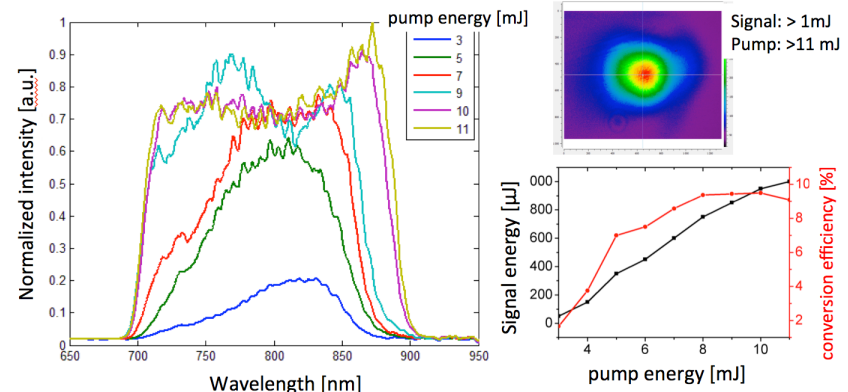

Figure 4: spectrum evolution (left) and efficiency (middle) versus pump energy of the OPCPA. A beam profile representing the beginning of the spatial distortion due to saturation is also represented (right).

The optimal operation point is then defined for $\sim 10 \%$ efficiency and an output energy of $0.95 \mathrm{~mJ}$. In fig. 5, the inputoutput spectrum of the OPCPA at this operation point is given (in linear and log scale). The excellent beam profile is also given (fig.5 right).
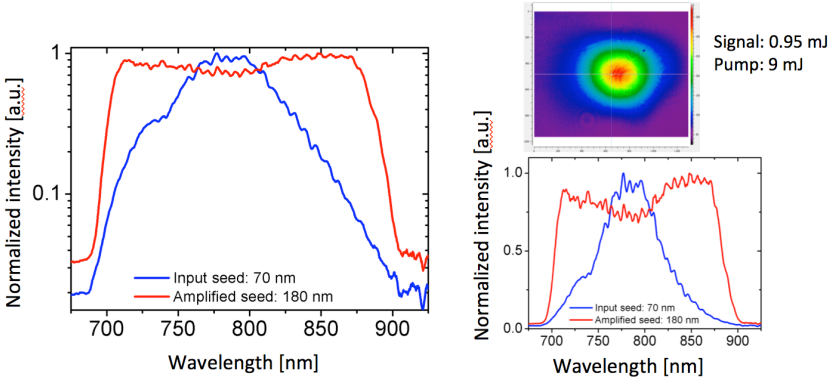

Figure 5: Spectra before and after the OPCPA in linear and log scale for an energy of $0.95 \mathrm{~mJ}$ (left-middle). Beam profile of the OPCPA at the same operation point (right).

For the temporal characterization of the OPCPA output, the amplified 6-ps-streched pulses have been compressed using the large Offner stretcher of the Apollon chain set close to the zero dispersion configuration. Using the stretcher in such a configuration gives us the possibility for a combined investigation: first the experimental evaluation of the AOPDF capacity for active compensation of the higher order spectral phase and optimization of the pulses contrast in the coherent range $(<1 \mathrm{ps})$ and, second, the study of the incoherent contrast taking into account the maximum of the potential contributions, including the Offner stretcher itself and more specifically the impact of its optical components quality.

The compressed pulses are first analyzed and optimized with the use of a Self-Referenced Spectral Interferometry (SRSI) Wizzler device (Fastlite). After correction of the residual phase with the online AOPDF, we measured 9.5 fs to be compared to 8.1 fs Fourier transform limited (FTL) duration (fig. 6). The advantage of measuring the pulses using the SRSI is to be able to access to the coherent contrast down to typically $10^{-5}$. The residual phase in our case is negligible allowing an excellent coherent contrast. Indeed, as shown in the log scale inset graph of fig. 6, the pulse temporal shape measured is very closed to the FTL one. The pulse shape is imposed mainly by the squareshape spectrum. It is very important to underline that the subsequent high-energy Ti:Sapphire amplifiers will filter the amplitude towards a less platykurtic shape inducing an apodization of the temporal satellites. However, the reduced coherent contrast of an OPCPA operating near the saturation regime $(\approx 10 \%)$, is not a serious restriction when used as a Ti:Sapphire injector. Nevertheless, the OPCPA saturation remains an issue operating above $10 \%$ of efficiency regarding the spatial considerations. Moreover, in this configuration, the OPCPA gain (x500) remains very low in order to avoid any super-fluorescence. Indeed, in simple relative intensity level measurements using a fast photodiode, this super-fluorescence level, even when blocking the signal injection, has been estimated to be less than $10^{8}$ times lower than the pulse itself.
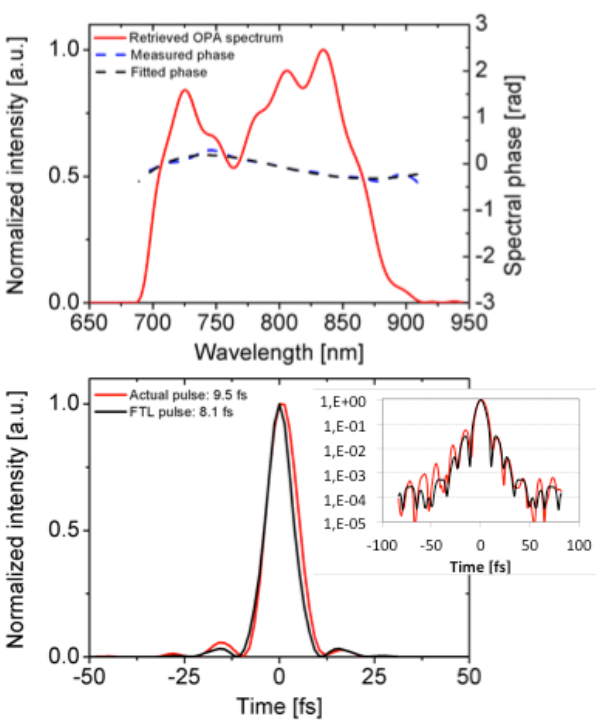

Figure 6: OPCPA compressed-pulse duration, SRSI measurement and coherent contrast: (top) spectrum, (bottom) temporal shape, (inset) $\log$ scale.

Even more important than the coherent contrast for PW system is the 100-ps-scale contrast. The incoherent contrast has been measured using a high dynamic third order autocorrelator. The measurement is shown in figure 8 indicating a floor noise with a mean contrast ratio of $\sim 10^{-12}$, limited by the dynamic range of the device. This guarantees an absence of parasitical peaks $>10^{-11}$ that could be hidden in the noise. Moreover the nature of the structures appearing at $-260 \mathrm{ps},-170 \mathrm{ps}$, $120 \mathrm{ps}$ and $-50 \mathrm{ps}$ are also probably due to noise. It is well known [19] that having higher dynamics with such apparatus is difficult. Taking into account the spectral bandwidth limitations of the non-linear crystal used in the autocorrelator (bandwidth limited for $100 \mathrm{fs}$ pulses) the peak power measured is in reality underestimated by one order of magnitude and therefore the contrast ratio can be safely estimated better than $10^{12}$ and closer to $10^{13}$. In fact, this conclusion is in agreement with the contrast evolution through the different stages of the source. The commercial $\mathrm{mJ}$ Ti:Sapphire amplifier contrast has been measured at $<10^{-8}$. The following XPW cleaning stage improves the contrast by at least $2-3$ orders of magnitude $\left(<10^{-10}\right)$ and finally the OPCPA gain $\left(\mathrm{x} 10^{3}\right)$ in the +-6 ps temporal window takes the contrast at least to the level of $10^{-13}$ based on the most conservative assumptions we could make.

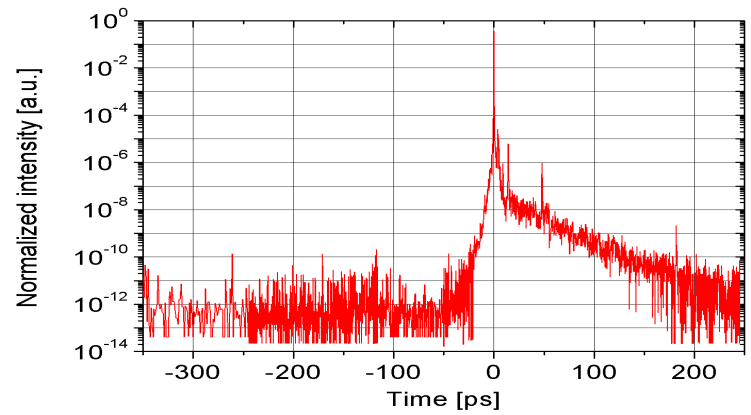

Figure 7: OPCPA compressed-pulse contrast measurement using a high dynamic third order autocorrelator. 
The ps-range contrast is also measured and is represented in figure 8. A pedestal extending to -30 ps is clearly visible. Since it is clearly longer than the OPCPA pump and since no super-fluorescence has been observed it is not due to the OPCPA (mainly according to the measurement made with the photodiode demonstrating CR better than $10^{8}$ ). The main contribution of the CR degradation in this time range is indeed ,as we will see, explained by another effect). In fact, we can attribute this contrast degradation to the stretcher (used in a compressor configuration) and more precisely to the convex mirror quality of the Offner telescope. Indeed, as predicted in [16,17], the roughness of this mirror where the spectrum is spatially dispersed can strongly impact the contrast.

The surface imperfections of this mirror act as a source of random fast spectral phase modulations which could lead to the degradation of the contrast of the pulses in the 10s ps range. We perform numerical simulations to estimate this effect. As input, we used the mirror rms roughness value of $1 \mathrm{~nm}$, specified by the supplier. Regarding the power spectral density (PSD) distribution, we assumed a typical exponential decay as in [16], corresponding to a normalized PSD in the time domain of $\exp (-|\mathrm{t}| / \mathrm{T})$ with $\mathrm{T}=2 \mathrm{ps}$. As shown in figure 8, we found a very good agreement with the experimental contrast trace. This contrast issues can be then improved working on the stretcher optical components quality and the spatial averaging effects due to the spatiotemporal aspect of the effect [16,17].

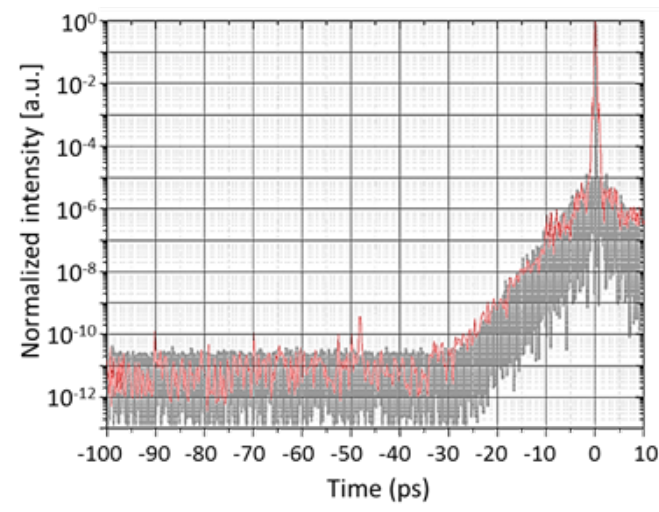

Figure 8: OPCPA compressed-pulse contrast in the 100 ps scale range. The thick grey line corresponds to the calculated contrast based on the model of [16].

In conclusion, we have presented a high-contrast injector for Ti:Sapphire high-energy amplifiers to be used in the Apollon 10PW laser. An unprecedented, to our best knowledge, complete contrast analysis has been performed with this source on different time scales fs, ps and sub-ns. Starting with a commercial Ti:Sapphire multipass amplifier producing $23 \mathrm{fs}$ pulses with a CR of $10^{-8}$, an XWP stage, pulse duration of $10 \mathrm{fs}$ has been demonstrated with a CR of $<10^{-10}$. After the OPCPA stage an estimated CR of $<10^{-13}$ is obtained for sub $10 \mathrm{fs}$ pulses. This injector demonstrates several advantages in the framework of a multi-PW laser. First, ultra-broadband amplification is demonstrated with a quasi-square shape compressible down to $9.5 \mathrm{fs}$. Moreover, the OPCPA efficiency is handled such as the beam profile maintains a very good quality, and the contrast is preserved from super-fluorescence. We have indeed seen that this optimization of the efficiency is crucial to preserve a good pulse quality and targeting efficiency higher than $10 \%$ means inevitably tradeoffs on this quality. Moreover, the pump of the OPCPA is based on DPSSL Yb-based technology, which allows the 100 $\mathrm{Hz}$ operation. This repetition rate of this seeder will be used advantageously to drive active pointing and synchronization stabilization servo-loops of the system between high-energy shoots. The next phase for the OPCPA system is to replace the OPCPA pump with a more energy-accurate pump based on a multipass $\mathrm{Yb}: \mathrm{CaF}_{2}$ amplifier $[12,13]$. This pump and the OPCPA stage are currently under commissioning phase at the Apollon laser facility. Finally, with a systematic contrast evolution analysis we were able to identify the technological weakest link, related to the stretcher optics quality responsible for the degradation of the ps-contrast of the generated pulses.

The authors gratefully acknowledge financial support from the ILEAPOLLON 07-CPER 017-01 contract.

\section{References}

[1] C. Danson, D. Hillier, N. Hopps, and D. Neely, High Power Laser Science and Engineering, 3, (2015)

[2] Tae Jun Yu, Seong Ku Lee, Jae Hee Sung, Jin Woo Yoon, Tae Moon Jeong, and Jongmin Lee, Opt. Express 20, 10807-10815 (2012)

[3] Z. Wang, C. Liu, Z. Shen, Q. Zhang, H. Teng, and Z. Wei," Opt. Lett. 36(16), 3194-3196 (2011).

[4]Yuxi Chu, Xiaoyan Liang, Lianghong Yu, Yi Xu, Lu Xu, Lin Ma, Xiaoming Lu, Yanqi Liu, Yuxin Leng, Ruxin Li, and Zhizhan Xu, Opt. Express 21, 29231-29239 (2013)

[5]M. Aoyama, K. Yamakawa, Y. Akahane, J. Ma, N. Inoue, H. Ueda, and H. Kiriyama, Opt. Lett. 28, 1594-1596 (2003)

[6] P. Antici, J. Fuchs, E. d'Humières, E. Lefebvre, M. Borghesi, E. Brambrink, C. A. Cecchetti, S. Gaillard, L. Romagnani, Y. Sentoku, T. Toncian, O. Willi, P. Audebert, and H. Pépin, Physics of Plasmas 14, 030701 (2007)

[7] J.P. Zou, C. Le Blanc, D.N. Papadopoulos, G. Chériaux, P. Georges, G. Mennerat, F. Druon, L. Lecherbourg, A. Pellegrina, P. Ramirez, F. Giambruno, A. Fréneaux, F. Leconte, D. Badarau, J.M. Boudenne, D. Fournet, T. Valloton, J.L. Paillard, J.L. Veray, M. Pina, P. Monot, J.P Chambaret, P. Martin, F. Mathieu, P. Audebert, F. Amiranoff, Special Issue on High Energy Density Physics and High Power Laser High Power Laser Science and Engineering, Volume 3, (January 2015 )

[8] D. N. Papadopoulos, J.P. Zou, C. Le Blanc, G. Chériaux, P. Georges, F. Druon, G. Mennerat, P. Ramirez, L. Martin, A. Fréneaux, A. Beluze, N. Lebas, P. Monot, F. Mathieu, P. Audebert, High Power Laser Science and Engineering, e34, 7 pages Vol. 4 (September 2016)

[9] A. Jullien, X. Chen, A. Ricci, J.P. Rousseau, R. Lopez-Martens, L.P. Ramirez, D. Papadopoulos, A. Pellegrina, F. Druon, P. Georges, Appl. Phys. B 102,4, 769-774 (2011)

[10] L.P. Ramirez, D. Papadopoulos, M. Hanna, A. Pellegrina, F. Friebel, P. Georges, and F. Druon, J. Opt. Soc. Am. B 30, 2607-2614 (2013)

[11] L.P. Ramirez, D.N. Papadopoulos, A. Pellegrina, P. Georges, F. Druon, P. Monot, A. Ricci, A. Jullien, X. Chen, J.P. Rousseau, and R. LopezMartens, Opt. Express 19, 93-98 (2011)

[12] J. Tümmler, R. Jung, H. Stiel, P. V. Nickles, and W. Sandner, Opt. Lett. 34, 1378-1380 (2009).

[13] I. N. Ross, P. Matousek, G. H. C. New, and K. Osvay, J. Opt. Soc. Am. B. 19(12), 2945-2956 (2002)

[14] J. Moses, S.-W. Huang, K.-H. Hong, O. D. Mücke, E. L. Falcão-Filho, A. Benedick, F. Ö. Ilday, A. Dergachev, J. A. Bolger, B. J. Eggleton, and F. X. Kärtner, Opt. Lett. 34, 1639-1641 (2009)

[15] C. Dorrer and J. Bromage Opt. Express 16 3058, (2008)

[16] J. Bromage, C. Dorrer and R. K. Jungquist, J. Opt. Soc. Am. B 29 1125, (2012)

[17] F. Friebel, A. Pellegrina, D.N. Papadopoulos, P. Camy, J. L. Doualan, R. Moncorgé, P. Georges, F. Druon, Appl. Phys. B 117, (2014).

[18] D. N. Papadopoulos, F. Friebel, A. Pellegrina, M. Hanna, P. Camy, J-L Doualan, R. Moncorge, P. Georges, F. Druon, Invited in Selected Topics in Quantum Electronics, IEEE Journal of Vol:21, Issue: 1 (2015)

[19] V. A. Schanz, F. Wagner, M. Roth, and V. Bagnoud, Opt. Express 25, 9252-9261 (2017) 